\title{
A Family Based Study on T-C Transition Polymorphism in Cyp17a1 Gene in Indian Children
}

Sukanya Gayan*, Abid Ali, Rajeev Kumar Pandey, Minu Bajpai

From International Conference on Human Genetics and 39th Annual Meeting of the Indian Society of Human Genetics (ISHG)

Ahmadabad, India. 23-25 January 2013

\section{Background}

Congenital adrenal hyperplasia (CAH) or 46, XX DSD is a result of a defect in the $\mathrm{P} 450$ adrenal enzymes responsible for the conversion of progesterones to glucocorticoids and mineralocorticoids. This syndrome affects both males and females but causes ambiguous genitalia only in females. Mutations in the CYP21 cause $90 \%$ of cases of CAH. The remainder of $\mathrm{CAH}$ cases is distributed among deficiencies of P450c11, CYP17 or steroid acute regulatory protein, depending on the ethnic origin of patients. Rarely, 46, XX, DSD can result from exposure to exogenous androgens such as those given in the past to prevent loss of pregnancy.

The present study was conducted to replicate a family based correlation of $\mathrm{C}$ to $\mathrm{T}$ transition polymorphism in Congenital adrenal hyperplasia (CAH).

\section{Materials and Methods}

A total of 60 samples (20 families) within a period of one year (October 2012 - 2013) associated with CAH, were collected from patient visiting the Out Patient Door (OPD) facility of the department of Paediatric Surgery, All India Institute of Medical Sciences (AIIMS). Genomic DNA was isolated from peripheral blood leukocytes of twenty patients and their family members using the phenol - chloroform method. Polymerase chain reactionrestriction fragment length polymorphism (PCR-RFLP) was used to detect the polymorphism in CYP17A1 using restriction enzyme, MSPA1.

Department of Pediatric Surgery, All India Institute of Medical Sciences, New Delhi, India 